\title{
Transmit Beamforming for Spectral Coexistence of Satellite and Terrestrial Networks
}

\author{
Shree Krishna Sharma, Symeon Chatzinotas, and Björn Ottersten \\ SnT - securityandtrust.lu, University of Luxembourg \\ Email: $\{$ shree.sharma, symeon.chatzinotas, bjorn.ottersten\}@uni.lu
}

\begin{abstract}
Herein, we study transmit beamforming techniques in an underlay cognitive mode for the coexistence of satellite and terrestrial networks with the satellite forward link as primary and the terrestrial downlink as secondary. Since geostationary satellite terminals have predetermined propagation characteristics so that they always point towards the geostationary satellite, the interference received by the satellite terminals from the terrestrial Base Station (BS) is confined in an angular sector. Based on this a priori knowledge, we propose transmit beamforming techniques at the BS to maximize the Signal to Interference plus Noise Ratio (SINR) towards the desired secondary user and to mitigate the interference towards the primary satellite terminals. Different types of Linearly Constrained Minimum Variance (LCMV) techniques have been proposed for our considered scenario where the exact locations and the number of satellite terminals within a specific angular sector are not known while designing the beamformer. Furthermore, an optimization problem is formulated for maximizing the Secondary User (SU) rate and it is shown that the worst case $S U$ rate depends on the Primary User (PU) distance, PU interference threshold and the angular separation of the desired $\mathrm{SU}$ from the region of interest.
\end{abstract}

Index Terms: Transmit Beamforming, Underlay, Cognitive Radio, Satellite-terrestrial Coexistence

\section{INTRODUCTION}

Recently, cognitive communications has been considered a promising technology in order to address the spectrum scarcity caused by the increasing demand of broadband and multimedia wireless services. This technique allows primary and secondary wireless systems to coexist within the same spectrum without affecting the normal operation of the primary systems. Wireless networks may exist within the same spectrum in different ways such as two terrestrial networks or two satellite networks or satellite-terrestrial networks. The most common cognitive techniques in the literature can be categorized into interweave or Spectrum Sensing (SS), underlay, overlay and database related techniques [1].

Existing spectrum sharing techniques mostly consider three signal dimensions i.e. frequency, time and area for sharing the available spectrum between primary and secondary systems. However, due to advancements in smart antennas and beamforming techniques, multiple users can be multiplexed into the same channel at the same time and in the same geographical area [2]. In the context of a Cognitive Radio (CR), angular dimension or directional dimension of spectral space can be considered as more efficient way of exploiting the space dimension to exploit the underutilized primary spectrum for the Secondary Users (SUs). Recently, the spatial dimension for spectrum sharing purpose has received important attention in the literature [2-4]. In [3], the angular dimension is used to detect the presence of a Primary User (PU) and to estimate the Direction of Arrival (DoA) of the PU signal. In [4], a directional SS scheme using a single radio switched beam antenna structure is proposed to enhance the sensing efficiency of a CR.

Beamforming is a signal processing technique used in antenna arrays with the advantages of spatial discrimination and spatial filtering capabilities [5]. Multi-antenna beamforming is an effective means to mitigate co-channel interference and has been widely used in traditional fixed spectrum based wireless systems [6-8]. In the context of a CR, beamforming techniques have been investigated for the secondary network for various objectives such as controlling interference [9], capacity maximization [10], Signal to Interference plus Noise Ratio (SINR) balancing [11]. The beamforming design problem in the context of an underlay $\mathrm{CR}$ is challenging since the underlay technique requires the interference caused by the SUs to be below the interference threshold level required by the PUs. In the existing CR literature, the beamforming techniques have been considered mostly in the coexistence scenario of two terrestrial networks [9-11]. In the context of cognitive satellite communications, SS techniques for dual polarized channels have been proposed in [12,13]. In [14], interference alignment technique has been proposed for spectral coexistence of monobeam and multibeam satellite systems. In [15], a receive beamformer has been proposed for the coexistence of satellite and terrestrial networks with both links operating in the normal reverse mode. In this paper, we apply the transmit beamforming techniques for spatial filtering for the spectral coexistence of satellite and terrestrial networks with the satellite forward link as primary and the terrestrial downlink as secondary. The main difference is that although the reception range of the satellite terminals is concentrated in an angular sector, we do not specifically know the number of interferers and the DoA of their signals.

Geostationary (GEO) satellites are located in the geosynchronous orbit above the equator and therefore transmit in a northerly direction if we consider the European continent. The GEO satellite terminals have therefore the special propagation characteristic to always point towards the GEO satellites (south). While considering the coexistence of a satellite network with the terrestrial cellular network, the reception 
range of all the satellite terminals is concentrated in an angular sector. Therefore, the interference provided by the Base Station (BS) to the satellite terminals depends on the directional properties of the transmitted beam designed at the BS. Furthermore, this interference becomes more prominent as we move towards the polar region from the equator due to lower elevation angles of the satellite terminals [1]. It can be noted that the interference from satellite to the terrestrial receiver is considered negligible due to different sensitivity levels of terrestrial and satellite receivers [16].

In this work, we propose different types of transmit beamforming techniques for the considered coexistence scenario. The proposed beamforming techniques can be implemented at the terrestrial BS to maximize the SINR towards the desired terrestrial user and to mitigate the interference towards the primary satellite terminals. The prior knowledge that all the GEO satellite terminals have certain angular reception range is the cognition that we exploit in this study. Since this is an inherent characteristic of SatComs, no interaction is needed between primary and secondary systems. One way of mitigating interference towards the PU terminals is by controlling the power of secondary transmission. However, the secondary rate has to be sacrificed while protecting the PU terminals. In this context, we formulate an optimization problem to maximize the SU rate by guaranteeing sufficient protection of PU terminals with a given transmit power budget.

The remainder of this paper is structured as follows: Section II presents the considered system and signal models. Section III describes the considered problem and proposes different transmit beamforming techniques in the context of the proposed scenario. Section IV provides the simulation environment and evaluates the performance of the proposed beamformers with the help of numerical results. Section V concludes the paper.

Notation: Throughout this paper, boldface upper and lower case letters are used to denote matrices and vectors respectively, $\mathbb{E}[\cdot]$ denotes expectation, $(\cdot)^{H}$ and $(\cdot)^{T}$ denote the conjugate transpose and transpose respectively.

\section{System And Signal Model}

We consider a practical coexistence scenario of satellite and terrestrial networks as shown in Fig. 1. We assume that both networks are operating in normal forward mode with the satellite link as primary and the terrestrial link as secondary i.e., satellite terminals are PUs and terrestrial terminals are SUs. In this context, a Fixed Satellite System (FSS) with the fixed satellite terminals (i.e., dishes) is considered to provide broadcasting services. From practical perspectives, the coexistence of terrestrial WiMax system and the FSS system operating in the $\mathrm{C}$ band (3.7-4.2 GHz, downlink) can be considered under this scenario. In this work, our main objective is to mitigate the interference from the terrestrial BS towards satellite terminals by applying transmit beamforming techniques at the terrestrial BS. Furthermore, we consider the situation of protecting satellite terminals located beyond the considered angular sector from the secondary interference

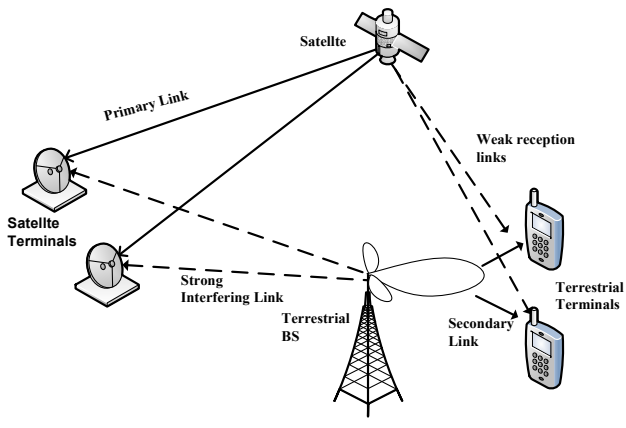

Fig. 1: Satellite terrestrial coexistence scenario

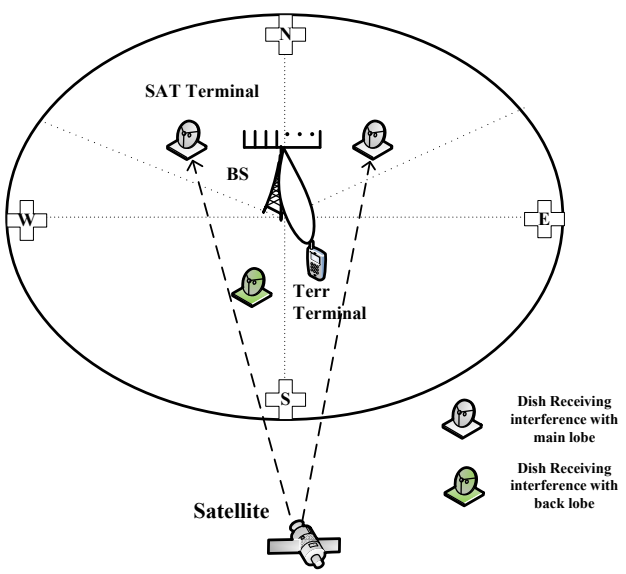

Fig. 2: Layout of the considered scenario (N,W,S and E denote North, West, South and East)

picked up by their backlobes. The layout of the considered scenario is shown in Fig. 2. The interference channel we are dealing in this scenario is the channel from terrestrial BS to the satellite terminals and the secondary channel is from terrestrial BS to the terrestrial terminals. By using some form of scheduling techniques, multiple terrestrial users can be supported under this system model.

For simplicity of analysis, we consider a single SU over a terrestrial link, multiple PUs within the considered sector of interest ${ }^{1}$ and one PU beyond this sector. Furthermore, we consider the SU and PU terminals to be equipped with a single antenna. Let $M$ be the number of antennas in the secondary BS antenna array and $K$ be the number of PUs in the considered sector. Let $s$ be a symbol which is to be transmitted from the secondary BS antenna at a particular time instant with $\mathbb{E}\left[s s^{H}\right]=1$ and $\mathbf{w}$ be the $M \times 1$ beamforming weight vector at the BS antenna array. Then the transmitted signal vector from the secondary BS antenna array can be written as: $\mathbf{x}_{s}=\mathbf{w} s$. The value of $\mathbf{w}$ can be written as: $\mathbf{w}=\sqrt{p} \mathbf{v}, p$ representing the power supplied to each antenna of the array and $\|\mathbf{v}\|=1$.

Let $\mathbf{h}_{p}$ be the channel vector from the BS to the satellite terminal i.e., PU and $\mathbf{h}_{s}$ be the channel vector from the BS to the terrestrial terminal i.e., SU. Then the received signal at

${ }^{1}$ The sector of interest is the considered angular sector in the northern part of the BS. 
the $\mathrm{SU}$ can be written as:

$$
y_{s}=\mathbf{h}_{s}^{H} \mathbf{x}_{s}+z_{s},
$$

where $\mathbf{h}_{s}$ is given by; $\mathbf{h}_{s}=\alpha_{s} \mathbf{a}\left(\theta_{s}\right)$, where $\mathbf{a}\left(\theta_{s}\right)$ is the array response vector with $\theta_{s}$ being a direction of arrival (DoA) for the SU signal, $\alpha_{s}$ is the path loss coefficient corresponding to the $\operatorname{DoA} \theta_{s}$ and $z_{s}$ is the independent and identically distributed (i.i.d.) Gaussian noise with zero mean and unit variance. The array response vector $\mathbf{a}(\theta)$ for a Uniform Linear Array (ULA) can be written as:

$$
\mathbf{a}(\theta)=\left[1, e^{\frac{-j 2 \pi d \sin (\theta)}{\lambda}}, \ldots, e^{\frac{-j 2 \pi(M-1) d \sin (\theta)}{\lambda}}\right]^{T}
$$

Similarly, the interfering signal at the PU terminal can be written as:

$$
y_{p}=\mathbf{h}_{p}^{H} \mathbf{x}_{s}+z_{s},
$$

where $\mathbf{h}_{p}$ is given by; $\mathbf{h}_{p}=\alpha_{p} \mathbf{a}\left(\theta_{p}\right)$, where $\mathbf{a}\left(\theta_{p}\right)$ represents the array response vector for DoA $\theta_{p}$ with $\theta_{p}$ being DoA for the PU signal and $\alpha_{p} \propto d_{p}^{-n}$ is the path loss coefficient between the secondary BS and the PU terminal with $d_{p}$ being the distance and $n$ being a path loss exponent.

\section{Proposed Transmit Beamforming Techniques}

Based on the system model defined in Section II, we try to address the following problems in this work.

1) How to mitigate the interference towards a certain angular sector based on the a priori knowledge of the propagation characteristics of GEO satellite terminals ? The beamforming weights at the BS should be designed in such a way that the transmitted power towards this angular sector is minimized.

2) Another problem is to design beamforming weights such that the SINR towards the desired SU is maximized. In other words, the SUs also should maximize the utilization of cognitive transmission.

3) Furthermore, it may be the case that the satellite terminals located beyond the sector of interest may receive the interfering signal from their backlobes. This may hamper the operation of the primary system. To solve this problem, we need to ensure that the interfering signal strength picked up by the backlobe of the satellite terminal is below the interference threshold level of the terminal.

4) Problem (3) can be solved by controlling transmitted power at the BS. However, this may affect the SU rate. This leads to defining and solving an optimization problem which we describe in the next section.

To address these problems, we propose three different techniques in the following section.

\section{A. Proposed Techniques}

1) Scaled LCMV Technique: In the standard LCMV beamformer, the weights are chosen to minimize the output variance or power subject to the response constraints [17]. To allow the transmitted signal towards the desired user's direction $\theta$ with response $g$, the weight vector can be linearly constrained in such a way that $\mathbf{w}^{H} \mathbf{a}(\theta)=g$, where $g$ is a complex constant [5]. Similarly, the transmissions towards the sector of interest can be minimized by choosing the weights in such a way that the output power or variance i.e. $\mathbf{w}^{H} \mathbf{R}_{d} \mathbf{w}$ is minimized with $\mathbf{R}_{d}$ being a $M \times M$ downlink spatial covariance matrix [17]. We assume that $\mathbf{R}_{d}$ is perfectly known ${ }^{2}$ while designing the beamformer. In this paper, we calculate it based on the knowledge of the array response vectors of the desired SU and the PU terminals ${ }^{3}$. To include the multiple constraints in the considered problem, the following constraint equation can be written: $\mathbf{C}^{H} \mathbf{w}=\mathbf{f}$, where $\mathbf{C}$ is a $M \times(K+1)$ constraint matrix, $\mathbf{f}$ is $L \times 1$ response vector, $L=K+1$ is the number of constraints. We consider the following constraint equation in our scenario:

$$
\left[\begin{array}{c}
\mathbf{a}^{H}\left(\theta_{1}\right) \\
\mathbf{a}^{H}\left(\theta_{2}\right) \\
\vdots \\
\mathbf{a}^{H}\left(\theta_{K+1}\right)
\end{array}\right]^{H} \mathbf{w}=\left[\begin{array}{c}
1 \\
0 \\
\vdots \\
0
\end{array}\right]
$$

Then the LCMV beamforming problem can be written as:

$$
\begin{array}{r}
\min _{\mathbf{w}} \mathbf{w}^{H} \mathbf{R}_{d} \mathbf{w} \\
\text { subject to } \mathbf{C}^{H} \mathbf{w}=\mathbf{f}
\end{array}
$$

The solution of the above problem can be written as [19]:

$$
\mathbf{w}_{\mathrm{LCMV}}=\mathbf{R}_{d}^{-1} \mathbf{C}\left(\mathbf{C}^{H} \mathbf{R}_{d}^{-1} \mathbf{C}\right)^{-1} \mathbf{f}
$$

In the proposed coexistence scenario, it is assumed that the angular sector in which the geostationary satellite terminals facing south are located is known to the beamformer but the exact locations and the number of PUs are not known to the beamformer. Since the LCMV technique requires discrete DoA values of the PUs, we uniformly sample the considered angular range in the interval of $\theta_{i}=\Delta / K$, where $\Delta=\theta_{\max }-\theta_{\min }$, $\theta_{\max }$ and $\theta_{\min }$ being the maximum and minimum values of the considered range. We then place one PU in each quantized angle and calculate the beamforming weights based on this set up using (6). We can then use these beamforming weights to study the performance of the LCMV beamformer in the proposed scenario. If the primary satellite terminals are present beyond the sector of interest, the back lobe of the terminals may pick up the interference power transmitted from the BS. To protect the PUs from this interference with a certain threshold, we can design a scaled LCMV beamformer by sacrificing some amount of transmit power in the desired direction. For the scaled LCMV, the weights of the LCMV beamformer given by (6) can be scaled as: $\mathbf{w}_{\mathrm{LCMVs}}=\epsilon * \mathbf{w}_{\mathrm{LCMV}}, \epsilon$ being a scaling parameter. The value of $\epsilon$ may range from a nonzero small positive value to 1 . When $\epsilon=0$, the beamformer response to all the directions becomes zero and therefore, the value of $\epsilon$ should be greater than zero. It can be noted that

\footnotetext{
${ }^{2}$ In practice, the downlink covariance matrix can be calculated from uplink covariance matrix by using different transformation approaches [18].

${ }^{3}$ To have the knowledge of DoAs of the PUs while designing the beamforming weights, we quantize the known angular sector in the uniform interval as described later in the following paragraph.
} 
the transmit power sacrifice in the desired direction increases with the decrease in value of $\epsilon$.

2) Modified LCMV Technique: In the standard LCMV technique, the response constraints towards the PUs are set as zeros and the response constraint towards the desired user is set as 1. In this scenario, the PUs are assumed to be located within an angular sector and the BS designs its beam pattern to mitigate the interference towards this sector. To consider the scenario of protection towards the backlobes of the PUs, we modify the standard LCMV optimization by putting one more constraint and formulate a new optimization problem. The new constraint is set in such a way that the interference picked up by the backlobe of the satellite terminals is below the interference threshold of the terminal. Let $I_{T}$ be the interference threshold set by the designers for the satellite terminals from the perspective of the interference picked up by the backlobe. It can be noted that as long as the interference picked by the backlobe of the terminal is below this level, there is no disturbance in the normal operation of primary system by the existence of secondary systems within the same spectrum.

The modified LCMV optimization problem is written as:

$$
\begin{array}{r}
\min _{\mathbf{w}} \mathbf{w}^{H} \mathbf{R}_{\mathbf{d}} \mathbf{w} \\
\text { subject to } \mathbf{C}^{H} \mathbf{w}=\mathbf{f} \\
\mathbf{w}^{H} \mathbf{R}_{\mathbf{p}} \mathbf{w} \leq I_{T},
\end{array}
$$

where $\mathbf{R}_{p}=\left\{\mathbf{a}\left(\theta_{\mathbf{b}}\right)\right\}\left\{\mathbf{a}^{\mathbf{H}}\left(\theta_{\mathbf{b}}\right)\right\}$ is the matrix containing the response vector towards the PU at the DoA of $\theta_{b}$ located beyond the sector of interest. Using Lagrangian multiplier method for solving the optimization problem (7), the Lagrangian can be written as:

$L(\mathbf{w}, \lambda, \eta)=\mathbf{w}^{H} \mathbf{R}_{d} \mathbf{w}+\lambda\left(\mathbf{C}^{H} \mathbf{w}-\mathbf{f}\right)+\eta\left(\mathbf{w}^{H} \mathbf{R}_{p} \mathbf{w}-I_{T}\right)=0$.

After differentiating the above Lagrangian function with respect to $\mathbf{w}^{H}$ and making equal to zero, the value of $\mathbf{w}$ can be written as:

$$
\mathbf{w}=-\lambda \mathbf{C}\left(\mathbf{R}_{d}+\eta \mathbf{R}_{p}\right)^{-1} .
$$

Substituting the value of $\mathbf{w}$ from (9) in the 1 st constraint of optimization problem (7), the value of $\lambda$ can be written as:

$$
\lambda=-\left(\mathbf{C}^{H}\left(\mathbf{R}_{d}+\eta \mathbf{R}_{p}\right)^{-1} \mathbf{C}\right)^{-1} \mathbf{f} .
$$

From (9) and (10), the value of $\mathbf{w}$ can be written as:

$$
\mathbf{w}=\left(\mathbf{R}_{d}+\eta \mathbf{R}_{p}\right)^{-1} \mathbf{C}\left[\mathbf{C}^{H}\left(\mathbf{R}_{d}+\eta \mathbf{R}_{p}\right)^{-1} \mathbf{C}\right]^{-1} \mathbf{f} .
$$

The above solution presents the value of $\mathbf{w}$ in terms of Lagrangian multiplier $\eta$. Furthermore, the complementary slackness condition for inequality constraint can be written as:

$$
\eta\left(\mathbf{w}^{H} \mathbf{R}_{p} \mathbf{w}-I_{T}\right)=0 .
$$

If $\eta=0$, the solution (11) reduces to the solution of standard LCMV optimization problem given by (6). If $\eta \neq 0$, the following condition should be satisfied

$$
\mathbf{w}^{H} \mathbf{R}_{p} \mathbf{w}-I_{T}=0 \text {. }
$$

The optimal value of $\mathbf{w}$ can be found using (13) and (11) but the process involves complex steps. Therefore, based on above derived expressions, we solve the problem (7) using a simple iterative algorithm. The iterative algorithm is given below.

An iterative algorithm for solving optimization
problem (7)
1) Initialize $\eta=0$ and calculate the value of $\mathbf{w}$ using
standard LCMV solution (6).
2) Check whether the second constraint $\mathbf{w}^{H} \mathbf{R}_{p} \mathbf{w} \leq I_{T}$
of problem (7) is satisfied or not. If the condition is
satisfied, proceed with step 5, otherwise follow step 3 .
3) Increment the value of $\eta$ by the value of step size $\Delta$ and
calculate the new value of $\mathbf{w}$ by substituting $\eta_{i+1} \longrightarrow$
$\eta_{i}+\Delta$ in (11) and the check the condition with step 2 .
4) Repeat steps 3 and 2 until the desired condition is
satisfied.
5) Use the calculated value of $\mathbf{w}$ for evaluating the perfor-
mance of beamformer in the considered scenario.

\section{B. SU Rate Maximization}

Let us denote the transmit signal covariance matrix by $\mathbf{R}_{t}$, which can be defined as

$$
\mathbf{R}_{t}=\mathbb{E}\left[\mathbf{x}_{s} \mathbf{x}_{s}^{H}\right]=p \mathbf{v} \mathbf{v}^{H}=\mathbf{w} \mathbf{w}^{H}
$$

The optimization problem for maximizing the rate of SUs by allowing the sufficient protection for the primary user can be written as:

$$
\begin{array}{r}
\max _{p \geq 0\|\mathbf{v}\|=1} \log \left(1+\operatorname{SINR}\left(\theta_{s}, p, d_{s}\right)\right) \\
\text { subject to } \Sigma p_{i} \leq P_{T}, i=1, \ldots, M \\
I_{p}\left(\theta_{p}^{(j)}, p, d_{p}\right) \leq I_{T H}, j=1, \ldots, K
\end{array}
$$

where $\operatorname{SINR}\left(\theta_{s}, p, d_{s}\right)$ represents the SINR of the desired SU and it is a function of $\theta_{s}$, transmit power across each antenna $p$, and the distance $d_{s}$ between the BS and the desired SU, $P_{T}$ is the total power budget. Furthermore, $I_{p}\left(\theta_{p}^{(j)}, p, d_{p}\right)$ is the interference received at the $j$-th PU due to secondary transmission and it is a function of $\theta_{p}, \mathrm{p}$, and the distance $d_{p}$ between the BS and the PU, $I_{T H}$ is the interference threshold required by the PUs.

The SINR for the desired SU considering the case of a single BS with uniform power allocation across multiple antennas can be written as:

$\operatorname{SINR}\left(\theta_{s}, p, d_{s}\right)=\mathbf{h}_{s}^{H} \mathbf{R}_{t} \mathbf{h}_{s}=\frac{p \lambda^{2} d_{s}^{-n}}{(4 \pi)^{2}}\left\{\mathbf{a}^{H}\left(\theta_{s}\right) \mathbf{v} \mathbf{v}^{H} \mathbf{a}\left(\theta_{s}\right)\right\}$,

where $\lambda$ is the wavelength of electromagnetic signal. Similarly, the interference received at the primary user due to secondary transmission can be written as:

$$
I\left(\theta_{p}^{(j)}, p, d_{p}\right)=\mathbf{h}_{p}^{H} \mathbf{R}_{t} \mathbf{h}_{p}=\frac{p \lambda^{2} d_{p}^{-n}}{(4 \pi)^{2}}\left\{\mathbf{a}^{H}\left(\theta_{p}^{(j)}\right) \mathbf{v} \mathbf{v}^{H} \mathbf{a}\left(\theta_{p}^{(j)}\right)\right\} .
$$

Using (16) and (17), the optimization problem in (17) can be 
written as:

$$
\begin{array}{r}
\max _{p \geq 0\|\mathbf{v}\|=1} \log \left(1+\alpha_{s}^{2} p\left\{\mathbf{a}^{H}\left(\theta_{s}\right) \mathbf{v} \mathbf{v}^{H} \mathbf{a}\left(\theta_{s}\right)\right\}\right) \\
\text { subject to } \Sigma p_{i} \leq P_{T}, i=1, \ldots, M \\
\frac{p \lambda^{2}}{\left(4 \pi d_{p}\right)^{2}}\left\{\mathbf{a}^{H}\left(\theta_{p}^{(j)}\right) \mathbf{v} \mathbf{v}^{H} \mathbf{a}\left(\theta_{p}^{(j)}\right)\right\} \leq I_{T H}, j=1, \ldots, K
\end{array}
$$

To solve the above optimization problem, firstly, we convert into a simple form as described below. Maximizing the term $\log \left(1+\alpha_{s}^{2} p\left(\mathbf{a}^{H}\left(\theta_{s}\right) \mathbf{v} \mathbf{v}^{H} \mathbf{a}\left(\theta_{s}\right)\right)\right.$ is equivalent to maximizing $\left|\sqrt{p} \mathbf{a}^{H}\left(\theta_{s}\right) \mathbf{v}\right|$. Since $\mathbf{w}=\sqrt{p} \mathbf{v}$, the objective function can be written as: $\left|\mathbf{a}^{H}\left(\theta_{s}\right) \mathbf{w}\right|$. Similarly, the interference power to the PU can be written as: $\alpha_{p}^{2}\left|\mathbf{a}^{H}\left(\theta_{p}\right) \mathbf{w}\right|^{2}$. Furthermore, we design $\mathbf{w}$ in such a way that the term $\mathbf{a}^{H}\left(\theta_{s}\right) \mathbf{w}$ has real value without loss of any generality. Therefore, the optimization problem in (18) after including additional constraint for the PU located beyond the considered sector can be written as:

$$
\begin{array}{r}
\max _{\mathbf{w}} \operatorname{Re}\left[\mathbf{a}^{H}\left(\theta_{s}\right) \mathbf{w}\right] \\
\text { subject to }\|\mathbf{w}\| \leq \sqrt{P_{T}} \\
\operatorname{Im}\left[\mathbf{a}^{H}\left(\theta_{s}\right) \mathbf{w}\right]=0 \\
\left|\alpha_{b} \mathbf{a}^{H}\left(\theta_{b}\right) \mathbf{w}\right| \leq \sqrt{I_{T}} \\
\left|\alpha_{p} \mathbf{a}^{H}\left(\theta_{p}^{(j)}\right) \mathbf{w}\right| \leq \sqrt{I_{T H}}, j=1, \ldots, K
\end{array}
$$

The above optimization problem is in the form of Second Order Cone Programming (SOCP) problem [20] and can be solved using standard convex optimization software CVX [21].

\section{NumericAl Results}

Let us consider a geographic sector which lies in the angular range from $10^{\circ}$ to $85^{\circ}$ with reference to the secondary BS. All the geostationary satellite terminals located in this sector face south (with respect to the position of the BS) for communicating with the geostationary satellite. We consider a single desired user at an angle of $-30^{\circ}$ and a ULA at the BS with the layout shown in Fig. 2. Furthermore, we consider a single satellite terminal at an angle of $-15^{\circ}$ to analyze the effect of secondary transmission on the backlobe of the satellite terminal. The simulation and link budget parameters for both the links (i.e., link between the BS and SAT terminal and the link between the BS and terrestrial terminal) are provided in Table I. To design a LCMV beamformer, we need the DoAs of the PUs. For this purpose, we quantize the considered angular sector in the interval of $5^{\circ}$ and consider one terminal in each quantized angle as mentioned in Section III-A.

Figure 3 shows the beam patterns of the standard LCMV, scaled LCMV, modified LCMV and the SU rate maximization approach. For the scaled LCMV technique, the scaling parameter $\epsilon=0.1$ was considered. From the figure, it can be noted that the beam pattern for scaled LCMV has a gain of $20 \mathrm{~dB}$ below the beam pattern for standard LCMV for all the considered angular range. In this way, we can reduce the transmitted signal towards the backlobe of the PU terminal by $20 \mathrm{~dB}$ with the sacrifice of $20 \mathrm{~dB}$ transmit power in the desired direction. This method may be suitable for terrestrial systems with terminals having higher sensitivity and for satellite systems with terminals having higher front to back ratio. The beamforming weights for the standard LCMV were computed using (6) and for the modified beamformer using the algorithm presented in Section III. Furthermore, the beamforming weights for the SU rate maximization approach were obtained by solving optimization problem (19) using CVX software [21]. The interference threshold towards the backlobe of the PU terminal $\left(I_{T}\right)$ located at $-15^{\circ}$ was set to be $-50 \mathrm{~dB}$ and the interference threshold towards the PU terminals $\left(I_{T H}\right)^{4}$ located in the considered angular region was set as $-80 \mathrm{~dB}$.

Figure 4 shows the performance comparison of modified LCMV and the standard LCMV beamformers in terms of the SINR. The beamforming weights calculated as described above were applied in the considered simulation environment where the exact positions and number of the PU terminals were unknown to the beamformer. During the simulation, the value of $I_{T}$ was considered to be $80 \mathrm{~dB}$ less than the power transmitted in the desired direction. From the figure, it can be noted that modified beamformer reduces the SINR towards the direction of the satellite terminal located at DoA of $-15^{\circ}$, thus protecting the satellite terminal from secondary interference. The reduced value of the SINR in the direction of the primary satellite terminal depends on the choice of the parameter $I_{T}$. This parameter should be chosen so as to meet the permissible interference level picked up by the backlobe of the satellite terminal in practical scenarios.

In the SU rate maximization approach, the transmit power in the desired direction depends on the chosen power threshold constraint in the direction of the PU terminals. To evaluate the performance of beamformer's response in the desired direction with respect to the change in the power threshold, simulations were carried by varying power threshold from $-50 \mathrm{dBW}$ to $0 \mathrm{dBW}$ in the DoAs of the PUs. For this purpose, the PU terminals were considered within the angular sector from $45^{\circ}$ to $85^{\circ}$ with each terminal at $5^{\circ}$ interval. Figure 5 presents the plot of transmitted power in the desired direction versus power threshold in the PU's direction. Furthermore, different plots have been presented considering desired users in different angular positions $\left(30^{\circ}, 20^{\circ}, 10^{\circ}, 0^{\circ}\right)$. It can be noted that the transmit power in the desired user's direction is the maximum when the constrained threshold power is kept at $-10 \mathrm{dBW}$ for all the cases. Furthermore, it can be noted that the transmit power in the desired direction increases as the angular difference between the desired SU and the considered sector becomes large (i.e., maximum at $0^{\circ}$ in Fig. 5).

To evaluate the performance of the beamformer with respect to the distance of the PU terminal from the BS, simulations were carried out considering the interference threshold of

\footnotetext{
${ }^{4}$ It should be noted that the response constraint towards these PU terminals in case of LCMV based approaches is zero.
} 
$-150 \mathrm{dBW}$. In this simulation settings, we include the path loss effect with path loss exponent $n=2$ in the optimization problem and find the optimal value of the beamformer's response. Figure 6 shows the worst case SU rate versus PU distance from the BS. The distance of the PU was varied from $0.5 \mathrm{~km}$ to $10 \mathrm{~km}$, transmit power constraint was considered to be $20 \mathrm{~W}$ and the desired user was considered at $-30^{\circ}$. From the figure, it can be noted that the SU rate increases with the increase in the PU distance. The rate of increase is fast at the lower values of the distance and slow at the higher values of the distance.

To show the overall effect of PU distance from the BS and the angular deviation from the considered sector, we have presented a three dimensional plot in Fig. 7. The distance range is considered from $0.5 \mathrm{~km}$ to $5 \mathrm{~km}$ and the angular deviation range was considered from $5^{\circ}$ to $30^{\circ}$ i.e., the DoAs of the SUs were considered in the range from $40^{\circ}$ to $15^{\circ}$. The interference power threshold at the PU terminal ${ }^{5}$ was considered to be $150 \mathrm{dBW}$. The SU rate was calculated by considering the worst case placement of the SU i.e., at a distance of $5 \mathrm{~km}$ from the BS. As the interference threshold towards the PU is decreased, the beamformer has to reduce its transmitted power and in turn the secondary rate is reduced. To show this effect with the help of optimization problem in (19), we have plotted the SU rate versus interference threshold at the PU terminal in Fig. 8. For this purpose, the interference threshold at the PU terminal is increased from $-200 \mathrm{dBW}$ to $-80 \mathrm{dBW}$. The power budget constraint was considered to be $20 \mathrm{~W}$ and the worst case SU distance was taken as $5 \mathrm{~km}$ from the BS.

While comparing the LCMV approaches with the SU rate maximization approach from Fig. 3, it can be noted that the later technique can provide slightly higher transmit power in the desired direction while the LCMV techniques can create very low interference towards the PU terminals located in the region of interest. It can be noted that there is less flexibility of introducing additional constraints such as power budget, interference threshold etc. in the LCMV based approaches. Furthermore, another difficulty for LCMV approach lies in acquiring the downlink covariance matrix. In SU rate maximization approach, there is more flexibility of introducing new constraints although the SU rate is dependent on the PU distance, interference threshold as well as the angular deviation from the sector of interest. It can be deduced that the choice of a particular technique mainly depends on the required performance level, the flexibility of introducing new constraints and the complexity of the technique.

\section{COnClusions And Future Work}

In this paper, we have proposed a coexistence scenario of the satellite and terrestrial networks with the satellite link as the primary and the terrestrial link as the secondary. Different transmit beamforming techniques have been proposed in an underlay cognitive mode for maximizing the SINR towards the

\footnotetext{
${ }^{5}$ It should be noted that this is the maximum tolerable interference power at the PU terminal including the effect of path loss.
}

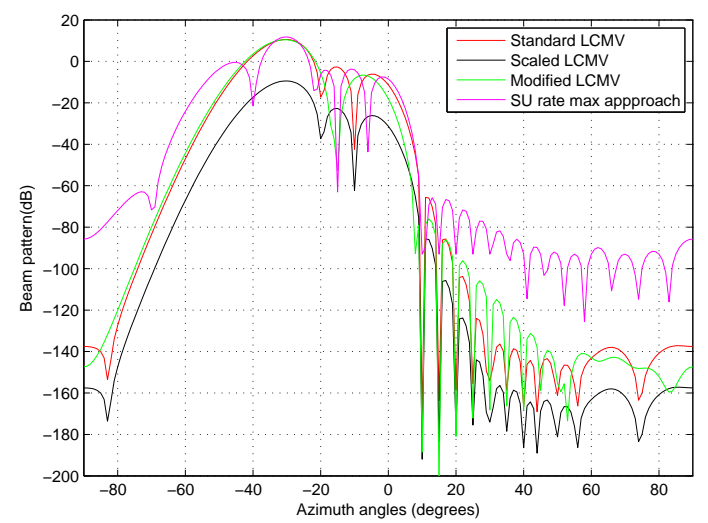

Fig. 3: Beam patterns of different transmit beamforming techniques

TABLE I: Simulation \& Link Budget Parameters

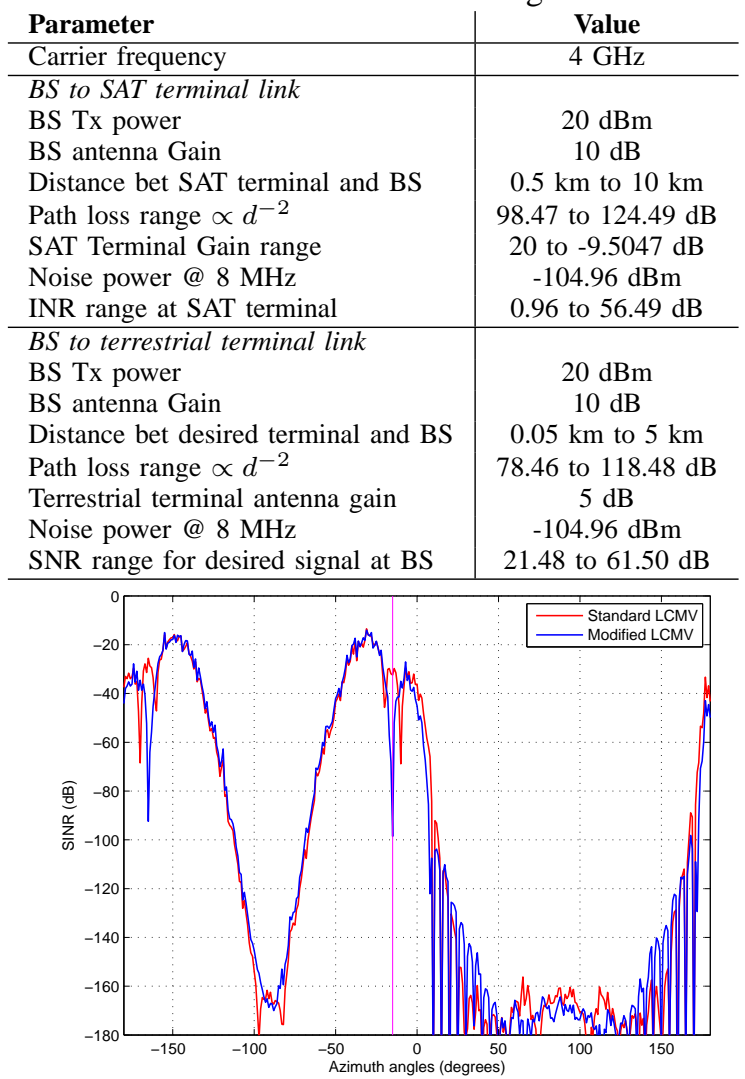

Fig. 4: SINR comparisons of the modified LCMV and standard LCMV in the considered scenario

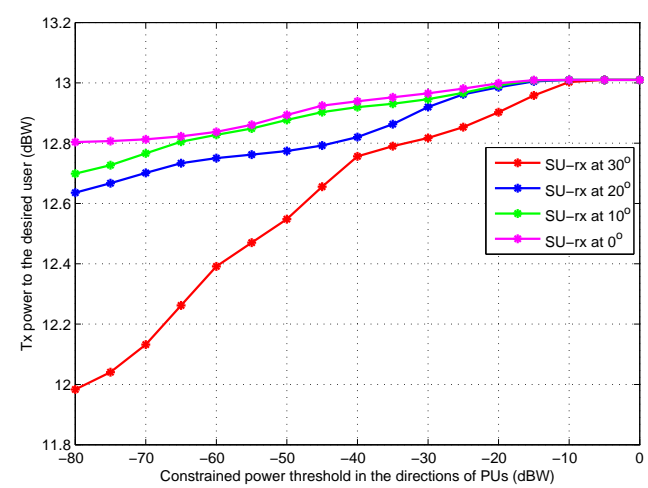

Fig. 5: Transmit power in the desired direction versus power threshold using optimization problem (19) 


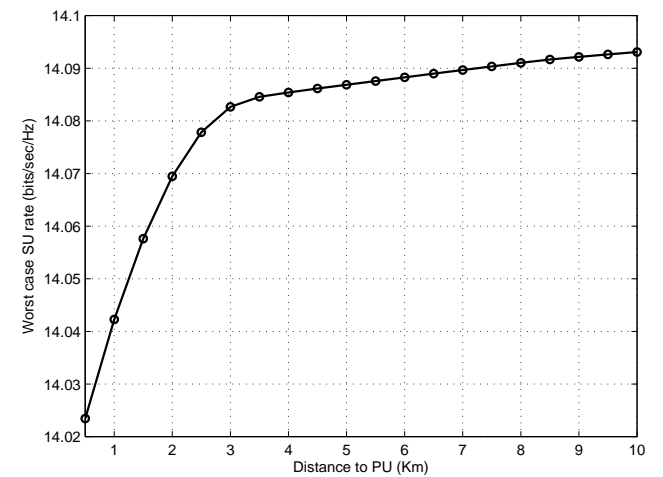

Fig. 6: Worst case SU rate versus PU distance from the BS

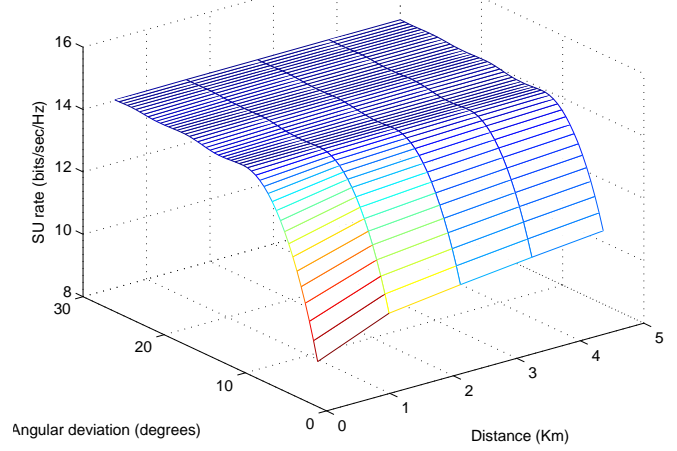

Fig. 7: Worst case SU rate versus PU distance and angular deviation from the sector of interest

desired SU and minimizing the interference towards the PUs. The choice of a technique in the considered scenario depends on the desired performance level as well as the flexibility of applying different constraints to the optimization problem. It can be concluded that the modified LCMV technique can provide the increased SINR towards the desired terminal and can mitigate the interference towards the specific angular sector by also providing sufficient protection towards the primary terminals located beyond the sector of interest. Furthermore, the considered SU rate maximization technique provides the flexibility of applying different constraints while maximizing the SU rate. It has been noted that the worst case SU rate is dependent on the PU distance, the permissible interference threshold at the PU terminals as well as the angular deviation

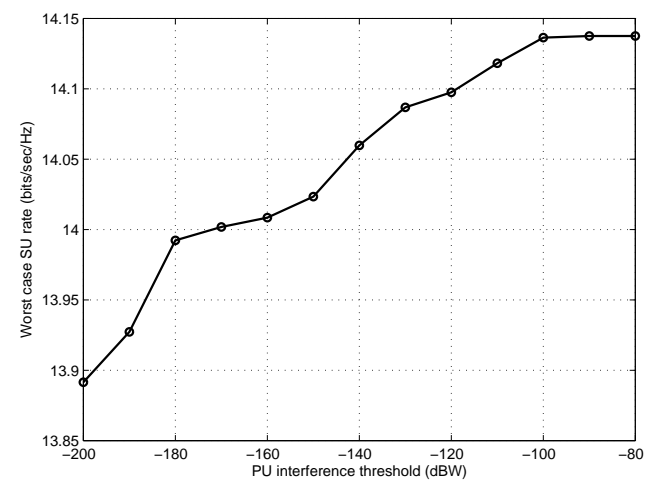

Fig. 8: Worst case SU rate versus interference threshold at the PU terminal of the desired user from the considered angular sector. We consider including robustness in the proposed techniques in the presence of angular uncertainty as our future work.

\section{ACKNOWLEDGEMENT}

This work was supported by the National Research Fund, Luxembourg under AFR (Aids Training-Research) grant for $\mathrm{PhD}$ project (Reference 3069102) on "Spectrum Sensing, Resource Allocation and Resource Management Strategies for Satellite Cognitive Communications", under the CORE project "CO2SAT: Cooperative and Cognitive Architectures for Satellite Networks".

\section{REFERENCES}

[1] S. K. Sharma, S. Chatzinotas, and B. Ottersten, "Satellite cognitive communications: Interference modeling and techniques selection," in 6th ASMS/SPSC Conf., Sept. 2012.

[2] H. Sarvanko, and et al, "Exploiting spatial dimension in cognitive radios and networks," in 6th Int. Conf. CROWNCOM, June 2011, pp. 360 -364.

[3] J. Xie, Z. Fu, and H. Xian, "Spectrum sensing based on estimation of direction of arrival," in Int. Conf. Computational Problem-Solving, Dec. 2010, pp. $39-42$.

[4] E. Tsakalaki, and et al, "Spectrum sensing using single-radio switchedbeam antenna systems," in 7th Int. Conf. CROWNCOM, June 2012.

[5] B. Van Veen and K. Buckley, "Beamforming: a versatile approach to spatial filtering," IEEE ASSP Mag., vol. 5, no. 2, pp. 4 -24, April 1988.

[6] F. Rashid-Farrokhi, K. Liu, and L. Tassiulas, "Transmit beamforming and power control for cellular wireless systems," IEEE J. Sel. Areas Commun., vol. 16, no. 8, pp. 1437 -1450, Oct. 1998.

[7] X. Wang and H. Poor, "Robust adaptive array for wireless communications," IEEE J. Sel. Areas Commun., vol. 16, no. 8, pp. $1352-1366$, Oct. 1998.

[8] V. Katkovnik, M.-S. Lee, and Y.-H. Kim, "Performance study of the minimax robust phased array for wireless communications," IEEE Trans. Commun., vol. 54, no. 4, pp. 608-613, April 2006.

[9] S. Yiu, M. Vu, and V. Tarokh, "Interference and noise reduction by beamforming in cognitive networks," IEEE Trans. Commun., vol. 57, no. 10 , pp. $3144-3153$, October 2009.

[10] T. Luan, F. Gao, X.-D. Zhang, J. Li, and M. Lei, "Rate maximization and beamforming design for relay-aided multiuser cognitive networks," IEEE Trans. Veh. Technol., vol. 61, no. 4, pp. 1940 -1945, May 2012.

[11] K. Cumanan, and et al, "SINR balancing technique for downlink beamforming in cognitive radio networks," IEEE Signal Process. Lett., vol. 17, no. 2, pp. $133-136$, Feb. 2010.

[12] S. K. Sharma, S. Chatzinotas, and B. Ottersten, "Exploiting Polarization for Spectrum Sensing in Cognitive SatComs," in Proc. 7th Int. Conf. CROWNCOM, June 2012.

[13] S. K. Sharma, S. Chatzinotas, and B. Ottersten, "Spectrum Sensing in Dual Polarized Fading Channels for Cognitive SatComs" in Proc. IEEE Globecom Conf., Dec. 2012.

[14] S. K. Sharma, S. Chatzinotas, and B. Ottersten, "Interference alignment for spectral coexistence of heterogeneous networks," EURASIP J. Wireless Commun. and Networking, vol. 46, 2013.

[15] S. K. Sharma, S. Chatzinotas, and B. Ottersten, "Spatial filtering for underlay cognitive SatComs," in Proc. Int. Conf. PSATS, June 2013.

[16] M. Hyhty, and et al, "Applicability of cognitive radio to satellite systems (ACROSS)," VIT technical Research centre, Tech. Rep., 2012, Finland.

[17] D. Z. Filho, C. C. Cavalcante, J. M. T. Romano, and L. S. Resende, "An LCMV-based approach for downlink beamforming in FDD systems in presence of angular spread," in Proc. EUSIPCO 2002, June 2002.

[18] B. Chalise, L. Haering, and A. Czylwik, "Robust uplink to downlink spatial covariance matrix transformation for downlink beamforming," in IEEE Int. Conf. Commun., vol. 5, June 2004, pp. 3010 - 3014 Vol.5.

[19] R. Lorenz and S. Boyd, "Robust minimum variance beamforming," IEEE Trans. Signal Process., vol. 53, no. 5, pp. 1684 - 1696, May 2005.

[20] W. Zhi, Y.-C. Liang, and M. Chia, "Robust transmit beamforming in cognitive radio networks," in IEEE Singapore Int. Conf. Commun. Systems, Nov. 2008, pp. $232-236$.

[21] S. B. M. Grant and Y. Ye, "CVX: Matlab software for disciplined convex programming," online, Nov 2007, www.stanford.edu./ boyd/cvx. 